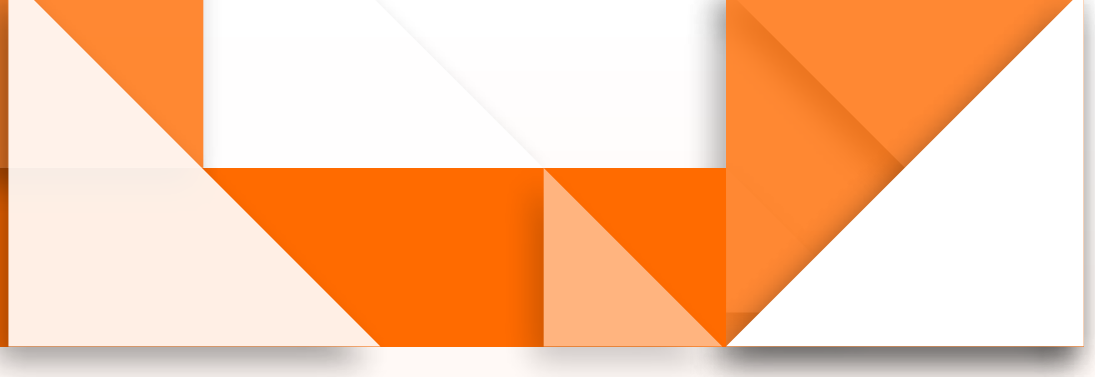

\section{1) Actividades prácticas de laboratorio e indagación en el aula}

\author{
- Atividades práticas de laboratório e indagação \\ na sala de aula \\ - Practical Activities in the Laboratory and \\ Inquiring in the Classroom
}

\section{Resumen}

Este artículo presenta una sistematización conceptual tomando aportes teóricos, definiciones y clasificaciones realizadas en el campo de la didáctica de las ciencias naturales sobre las actividades prácticas de laboratorio. Se pretende exponer el debate existente en la literatura científica sobre la utilidad de estas actividades y su influencia en la motivación del alumnado ya que algunos autores consideran que promueven el interés, pero no la motivación. Se presenta una serie de definiciones y características que permiten identificar a qué denominamos actividades prácticas de laboratorio y su diferencia con la idea de trabajo práctico de laboratorio. Se enumeran también las distintas clasificaciones según sus objetivos, planteadas desde diversas propuestas teóricas. Se introduce teoría sobre la indagación en el aula como propuesta para el abordaje de las actividades de laboratorio, en particular, el método de resolución de problemas por indagación (MRPI). Se plantea finalmente que las APL promueven procesos cognitivos que facilitan la comprensión, la problematización y la reflexión sobre el fenómeno estudiado.

Palabras clave

actividades prácticas; laboratorio; indagación científica; MRP
Nancy Edith Fernandez Marchesi ${ }^{*}$

Magister en Enseñanza de las Ciencias Experi-
mentales, profesora asociada exclusiva Instituto
de Educación y Conocimiento, Universidad
Nacional de Tierra del Fuego Ushuaia, Tierra
del Fuego, Argentina.
nfernandez@untdf.edu.ar
ORCID: http://orcid.org/0000-0001-5282-2012

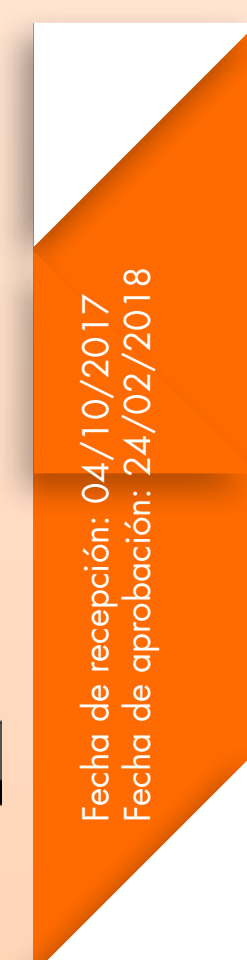




\section{Abstract}

This article presents a conceptual systematization based on theoretical contributions, definitions, and classifications made in the field of natural science teaching about practical activities in the lab. The goal is to explain the current debate in scientific literature on the usefulness of these activities and their influence in student motivation, since some authors believe they promote interest but not motivation. We present a series of definitions and characteristics that allow us to identify what we define as practical activities in the laboratory and their difference with the idea of practical work in the laboratory. We also list different classifications according to objectives, established from various theoretical proposals. The theory of inquiry in the classroom is introduced as a proposal to address laboratory activities, and particularly, the problem-solving inquiry method (PSIM). Lastly, it is proposed that LPAs promote cognitive processes that facilitate comprehension, problematization and reflection on the studied phenomenon.

Keywords

practical activities; laboratory; scientific inquiry

\section{Resumo}

Este artigo apresenta uma sistematização conceitual, baseada em contribuições teóricas, definições e classificações realizadas no campo da didática das ciências naturais sobre as atividades práticas de laboratório. Pretendemos expor o debate existente na literatura científica sobre a utilidade dessas atividades e sua influência na motivação dos estudantes, já que alguns autores consideram que promovem o interesse, mas não a motivação. Apresentamos uma série de definições e características que permitem identificar o que denominamos atividades práticas de laboratório e sua diferença com a ideia de trabalho prático de laboratório. Enumeramos, também, as diversas classificações segundo seus objetivos, colocadas desde diversas propostas teóricas. Introduzimos a teoria sobre a indagação na sala de aula como proposta para a abordagem de atividades de laboratório, especificamente, o método de resolução de problemas por indagação (MRPI). Proponemos, finalmente, que as APL promovem processos cognitivos que facilitam a compreensão, a problematização e a reflexão sobre o fenômeno estudado.

Palavras-chave

atividades práticas; laboratório; indagação científica 


\section{Introducción}

La investigación sobre la enseñanza de las actividades prácticas de laboratorio (APL) en biología tiene un importante desarrollo. Al respecto, varios autores (Abrahams, 2009; Abrahams, Reiss y Sharpe, 2014; Abrahams y Saglam, 2010; Abrahams y Sharpe, 2010; Barolli, Laburú y Guridi, 2010; Correa, Sánchez y Valbuena Ussa, 2007; Fernández-Marchesi, 2014; Hofstein y Mamlok-Naaman, 2007) ofrecen un estado del arte del laboratorio de ciencias. Estos trabajos dan cuenta del elevado porcentaje de artículos publicados con relación a las actividades experimentales de laboratorio en biología. En general, los artículos abordan dos dimensiones: la problematización de las APL en cuanto a la enseñanza de conceptos biológicos, y el diseño e implementación de propuestas didácticas que incluyen APL o protocolos de APL para la enseñanza de un concepto biológico específico.

A su vez, otros autores (Barberà y Valdés, 1996; Carrascosa, Gil Pérez y Vilches, 2006; García Barros, Mondelo Alonso y Martinez Losada, 1998; Hodson, 1994; Saraiva-Neves, Caballero Sahelices y Moreira, 2006) plantean que en diversas investigaciones gran parte de los trabajos experimentales que se realizan en las aulas son meramente ilustrativos, se reducen a experiencias tipo recetas, generan poca motivación y favorecen un número muy limitado de competencias. Mencionan que tal y como se implementan los trabajos prácticos de laboratorio son confusos, poco productivos y promueven pocos aprendizajes sobre ciencias. Sin embargo, a pesar de todos los aspectos negativos que explicitan estos investigadores, todos ellos destacan la importancia de las APL para la enseñanza de las ciencias e interpretan que dichos aspectos tienen relación con cómo se los usa y cómo se los diseña y no con la APL en sí.
En esta línea, numerosos autores (Chavez de Souza y Tauchen, 2015; Cortés Gracia y de la Gándara Gómez, 2006; Del Carmen, 2011 ; Fernández-Marchesi, 2014; García Barros et al., 1998; Hodson, 1994; Tamir y García Rovira, 1992) destacan la importancia de las actividades de laboratorio por su carácter motivacional del aprendizaje. Sin embargo, Abrahams (2009) destaca la diferencia entre motivación e interés, ya que si bien ciertas actividades prácticas pueden generar interés o compromiso en un contenido en particular, hay poca evidencia que sugiera que las actividades de laboratorio motiven a los alumnos hacia las ciencias naturales en general o hacia el estudio de una disciplina científica en particular.

El interés situacional es el que se estimula en un individuo como consecuencia de una situación didáctica particular, por ejemplo, cuando un alumno realiza una actividad de laboratorio. A diferencia del interés personal, este es susceptible a la influencia del profesor a corto plazo y, aunque es menos probable que persista con el tiempo, proporciona una oportunidad para que los profesores influyan positivamente en el aprendizaje de sus alumnos (Abrahams, 2009).

El debate es amplio y diverso, y a medida que se profundizan y amplían las investigaciones surgen nuevos indicios sobre la problemática de la enseñanza de las actividades de laboratorio.

\section{Las actividades prácticas de laboratorio. Evolución conceptual}

En la enseñanza de las ciencias naturales (biología, física y química) se pueden emplear muchos tipos de actividades, considerando esta como toda situación de enseñanza en la que hay interacción entre tareas del docente y tareas de los alumnos. Muchas son comunes con otras disciplinas, sin embargo, las actividades de 
laboratorio o las salidas de campo son características de las disciplinas científicas y en ellas predomina el enfoque investigador, siendo la fuente de información el fenómeno explorado (Cañal de León et al., 2011).

De la revisión de la literatura, se concluye que se toman como sinónimos los conceptos trabajos prácticos y actividades prácticas. Sin embargo, algunos autores (Bonito y Trindade, 2008; Fernández-Marchesi, 2014) han distinguido estos conceptos a partir de diferentes enfoques de análisis. La palabra trabajo' proviene de una idea ligada al sufrimiento o al dolor (Tripalium) ${ }^{2}$. Su origen está vinculado con un enfoque de enseñanza positivista en el que el aprendizaje se considera como un trabajo que requiere de esfuerzo, sufrimiento y sacrificio (Fernández-Marchesi, 2014). En cambio, el concepto actividad posee una connotación relacionada con la acción de obrar, de operar, de realizar tareas propias. Es un concepto apropiado para denominar las actividades que los estudiantes pueden realizar en un laboratorio desde un enfoque constructivista Bonito, 1996). Por ello, el concepto de actividades prácticas (AP) se vislumbra como más amplio y válido dentro de las corrientes actuales de la didáctica de las ciencias naturales.

\section{Características de las actividades prácticas de laboratorio}

Algunos autores (Barberà y Valdés, 1996; Del Carmen, 2011 ; Del Carmen et al., 2000)desarrollan ciertas ideas que permiten identificar a qué llamamos actividades prácticas de laboratorio:

- Las realizan los alumnos con un grado variable de participación en su diseño y ejecución.

- Implican el uso de procedimientos científicos de diferentes características (observación, formulación de hipótesis, realización de experimentos, técnicas manipulativas, elaboración de conclusiones, entre otros) con diferentes grados de aproximación con relación al nivel de los alumnos.

- Requieren del uso de un material específico, semejante al utilizado por los científicos, aunque a veces simplificado para facilitar su uso por los alumnos.

- Con frecuencia se realizan en un ambiente diferente al del aula, como por ejemplo el laboratorio o el campo, aunque también pueden llevarse a cabo en el aula.

- Presentan algunos riesgos debido a la manipulación de instrumentos.

- Son actividades más complejas de organizar que las de lápiz y papel 
Otros autores (Carrascosa et al., 2006) enumeran algunos requisitos que deben reunir estas actividades:

1. Presentar situaciones problemáticas abiertas adecuadas al nivel de los estudiantes

2. Favorecer la reflexión de los estudiantes, incluyendo las implicancias entre ciencia, tecnología, sociedad y ambiente (CTSA)

3. Potenciar los análisis cualitativos y formular preguntas sobre lo que se busca.

4. Plantear la emisión de hipótesis como actividad central de la investigación científica.

5. Conceder importancia a la elaboración de diseños y a la planificación de la actividad experimental por los propios estudiantes.

6. Plantear el análisis detenido de los resultados a la luz del cuerpo de conocimientos disponible.

7. Contemplar las implicaciones CTSA del estudio realizado

8. Dar importancia a la elaboración de informes científicos y resaltar el papel de la comunicación científica

9. Fomentar la dimensión colectiva del trabajo científico organizando equipos de trabajo

Otros (Del Carmen, 2011) señalan la importancia de este tipo de actividades:

- Desempeñan un papel fundamental en el incremento de la motivación hacia las ciencias experimentales.

- Son una ayuda inestimable para avanzar en la comprensión de los planteamientos teóricos de la ciencia y el desarrollo del razonamiento científico

- Facilitan la comprensión de cómo se elabora el conocimiento científico y de su significado.

- Son insustituibles para la enseñanza y el aprendizaje de procedimientos científicos y con las actitudes hacia la ciencia (curiosidad, confianza en los recursos propios, apertura hacia los demás, etc.).

No obstante, el tiempo dedicado a las actividades prácticas en la enseñanza de las ciencias naturales suele ser reducido (Nieda, 1994). Ello puede explicarse por diferentes motivos: excesivo número de alumnos, ausencia de instalaciones adecuadas o instrumentos específicos, o escasa formación docente (Fernández-Marchesi, Marcangeli y Romero, 2011 ). Sanmartí Puig (2002) también señala que muchos docentes opinan que la función de las actividades prácticas en el aprendizaje de conceptos no es esencial, o que el principal valor que se les da a estas actividades prácticas es el aumento en la motivación del alumnado, lo que lógicamente también se puede conseguir mediante otras estrategias.

Además de los motivos sistematizados en la literatura, surgen otros de tipo más subjetivo, ya que la realización de APL exige a los docentes dedicar tiempo para su preparación y afrontar y solucionar problemas que puedan presentarse en su aplicación. Esto requiere unas dosis altas de motivación por parte del profesorado, y un estímulo y apoyo de parte de la institución educativa (Del Carmen, 2011).

Muchos estudios muestran que las APL presentan distintos déficits. Hodson (1994) fue uno de los primeros que los recogió de forma sistemática y señaló que: 
- Las actividades se desarrollan sin base teórica.

- El contenido lo promueve el profesor, lo que limita la construcción personal de los significados por parte de los alumnos.

- Los estudiantes muchas veces son meros consumidores de planificaciones realizadas por el profesor.

- Los estudiantes a menudo no se apropian de la teoría adecuada para interpretar lo que observan, o solo realizan interpretaciones erróneas a la luz de concepciones erróneas.

Este autor plantea además serias reservas en cuanto a la eficacia de los trabajos prácticos de laboratorio como se presentan usualmente en las clases de ciencias. Afirma que sería de mucha utilidad redefinir la noción de actividad práctica de laboratorio para incluir una variedad mayor de estrategias, que posibilitara contemplar una mayor variedad de objetivos teniendo en cuenta las distinciones entre:

- Aprender ciencia: adquirir el conocimiento conceptual y teórico.

- Aprender sobre la ciencia: desarrollar una comprensión sobre la naturaleza de los métodos de la ciencia y una percepción de las complejas interacciones entre ciencia, tecnología, sociedad y ambiente (CTSA).

- Hacer ciencia: desarrollar habilidades en la investigación científica y la resolución de problemas.

\section{Objetivos de las actividades prácticas de laboratorio}

Con relación a los objetivos que persiguen las APL, numerosos autores (Caamaño, 2004, 2007; Leite y Figueiroa, 2004; Martins et al., 2007; Sanmartí Puig, 2002) han realizado diversas categorizaciones y/o clasificaciones.

Por un lado, Sanmartí Puig (2002) clasifica las APL por:

1. Tipo de preguntas o problemas planteados:

a. orientados a aprender procedimientos o técnicas

b. orientados a la observación sistémica de objetos, organismos o fenómenos

c. de tipo inductivo, en los que se solicita detectar regularidades o inferir relaciones entre variables.

d. de tipo deductivo, en los que se solicita que utilicen razonamientos deductivos para relacionar ideas generales o teorías con el fenómeno observado.

e. hipotético-deductivos, en los que se solicita identificar y combinar variables para comprobar una hipótesis. 
2. El grado de apertura

a. Nivel cero: Se le da al estudiante la pregunta, el método y los resultados.

b. Nivel uno: Se dan todos los apartados menos los resultados.

C. Nivel dos: se da solo la pregunta.

d. Nivel tres: se indica un fenómeno o una situación y a partir de ello debe formularse la pregunta, proponer el método, obtener resultados y deducir conclusiones.

Caamaño (2004, 2007), por otra parte, propone cuatro tipos de actividades prácticas: experiencias, experimentos ilustrativos, ejercicios prácticos e investigaciones.

- Las experiencias son actividades prácticas destinadas a obtener una familiarización perceptiva con los fenómenos.

- Los experimentos ilustrativos son actividades destinadas a interpretar un fenómeno, ilustrar un principio o mostrar una relación entre variables. Pueden constituir una aproximación cualitativa o cuantitativa al fenómeno. En el caso de ser realizadas únicamente por el profesor se acostumbran a denominar "demostraciones".

- Los ejercicios prácticos son actividades diseñadas para aprender determinados procedimientos o destrezas, o para realizar experimentos cuantitativos que ilustren o corroboren la teoría.

- Las investigaciones son actividades encaminadas a resolver un problema teórico o práctico mediante el diseño y la realización de un experimento y la evaluación del resultado.
Leite y Figueiroa (2004), a su vez, le aportan algunas diferencias a la clasificación propuesta por Aureli Caamaño (2004) y proponen la siguiente:

1. Aprendizaje de conocimiento procedimental

a. Ejercicios: Apuntan al desarrollo de destrezas (observación, medición, manipulación) y permiten el aprendizaje de técnicas de laboratorio. Requieren una descripción detallada del procedimiento y los más complejos pueden exigir una demostración.

2. Aprendizaje de conocimiento conceptual

a. Refuerzo de conocimiento conceptual

- Actividades para familiarizarse con los fenómenos: se basan en los sentidos y dan al alumno la oportunidad de oler, sentir, oír. No introducen ningún concepto nuevo, pero dan una noción del concepto o principio en cuestión.

- Actividades ilustrativas: confirman que el conocimiento presentado es verdadero. Se basan en la ejecución de un protocolo tipo receta, estructurado con el fin de conducir el resultado previamente conocido por los alumnos.

b. Construcción de conocimiento conceptual

- Actividades orientadas hacia la determinación de lo que ocurre: conducen la construcción de conocimientos nuevos mediante la implementación de una actividad detalladamente descrita en un protocolo que lleva a 
los alumnos a la obtención del resultado que se pretende y que ellos desconocían inicialmente.

- Investigaciones: conducen a la construcción de nuevos conocimientos conceptuales gracias a un procedimiento de resolución de problemas. Los alumnos tienen que encontrar una estrategia para resolver el problema, ponerla en práctica y además evaluarla y reformularla en caso necesario, integrando los anteriores tipos de actividades.

c. Reconstrucción de conocimiento conceptual

- Prevé-observa-explica-reflexiona (procedimiento presentado): promueve la reconstrucción de conocimiento de los alumnos empezando por confrontarlos con una cuestión que permite hacerlos conscientes de sus ideas previas para luego confrontarlas con los datos empíricos que permitan apoyarlas o debilitarlas. Existe un protocolo cuya implementación permite obtener los datos necesarios.

- Prevé-observa-explica-reflexiona (procedimiento por definir): promueve la reconstrucción de conocimiento de los alumnos empezando por confrontarlos con una cuestión que permite hacerles conscientes de sus ideas previas para luego confrontarlas con los datos empíricos que permitan apoyarlas o debilitarlas. Los alumnos tienen que encontrar una estrategia para poner a prueba sus ideas.

3. Aprendizaje de metodología científica

a. Investigaciones: dado que no están apoyadas por protocolos, las investigaciones permiten a los alumnos, además de la construcción de conocimientos conceptuales nuevos, el desarrollo de competencias de resolución de problemas y de la comprensión de los procesos de la ciencia y de la naturaleza.

Martins et al. (2007) citando a Leite (2001) proponen las siguientes denominaciones:

- Trabajo laboratorial (TL): se define como un conjunto de actividades que surge en el laboratorio, con equipos propios o con estos mismos equipos en otro lugar si eso no implica riesgo para la salud o seguridad.

- Trabajo práctico (TP): se aplica a todas las situaciones en las que el alumno participa activamente en la realización de una tarea que puede ser o no del tipo de laboratorio.

- Trabajo experimental (TE): se aplica a actividades prácticas donde hay manipulación de variables.

La figura 1 ilustra la relación entre los tres tipos de actividades: 


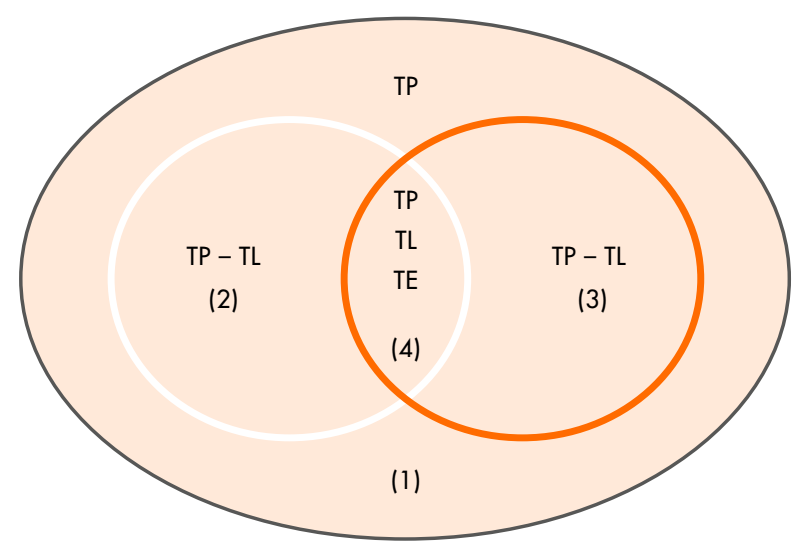

Figura 1. Tipos de trabajos prácticos según Martins et al. (2007)

En la zona (1) se encuentra el trabajo práctico que no es del tipo de laboratorio, ni del experimental (colección de hojas de árboles y arbustos para su posterior clasificación).

En la zona (2) surge el trabajo práctico de laboratorio que no es del tipo experimental (el aprendizaje de técnicas de laboratorio, filtración, una preparación para la observación con el microscopio).

En la zona (3) se encuentra el trabajo práctico experimental que no es del tipo laboratorial y, por lo tanto, no utiliza aparatos o instalaciones de laboratorio (experimentos sobre los factores que afectan el crecimiento de plantas en entornos naturales).

En la zona (4) se encuentran los trabajos prácticos de laboratorio experimentales, que operan con distintos grados de apertura para que los estudiantes encuentren respuestas a pregunta de investigación (Martins et al., 2007).

\section{La indagación científica como} enfoque propicio en el desarrollo de las APL

La indagación científica hace referencia a las diferentes formas en las que los científicos estudian el mundo natural y proponen explicaciones basadas en la evidencia derivada de su trabajo.
La indagación se refiere también a las actividades de los alumnos en las cuales desarrollan conocimiento y comprensión de las ideas científicas, así como el entendimiento de la forma en que los científicos estudian el mundo natural. Es el proceso intencional de diagnóstico de problemas, la crítica de los experimentos y distinción de alternativas, la planificación de las investigaciones, la investigación de conjeturas, la búsqueda de información, la construcción de modelos, el debate con los compañeros y la formación de argumentos coherentes (Linn, Davis y Bell, 2004). Esta definición caracteriza de forma amplia los componentes de la indagación, incluyendo la naturaleza y las capacidades para hacer indagación y la forma en que los científicos trabajan en el desarrollo del conocimiento científico

La indagación es un proceso intencional de diagnóstico de problemas, crítica de experimentos y distinción de alternativas, planificación de investigaciones, investigación de conjeturas, búsqueda de información, construcción de modelos, debate con compañeros y construcción de argumentos coherentes.

La mayoría de los autores, entre ellos Cortés Gracia y de la Gándara Gómez (2006) y Garritz (2010), señalan que el término indagación es una palabra polisémica y de moda 
en didáctica de las ciencias naturales y han enumerado las habilidades que es posible movilizar mediante la indagación científica.

Khan (2007) menciona que las actividades básicas de la indagación son: identificar un problema y reunir información; hacer predicciones; dar sentido a las observaciones y buscar patrones en la información; usar analogías e intuición para conceptualizar los fenómenos; analizar y representar datos; postular factores causales potenciales; trabajar con las pruebas para desarrollar y revisar las explicaciones; generar relaciones hipotéticas entre las variables; evaluar la consistencia empírica de la información; formular y manipular modelos mentales o físicos (modelado); coordinar los modelos teóricos con la información, y compartir lo que se ha aprendido durante la indagación con otras personas.

En un resumen de todas las actividades mencionadas por la literatura (Espinosa Bueno, Garritz Ruiz, Labastida Piña y Padilla, 2010; Espinosa Bueno, Labastida Piña, Padilla, y Garritz Ruiz, 2011 ; Garritz Ruiz, Labastida Piña, Padilla y Espinosa Bueno, 2009) las actividades que se llevan a cabo durante la indagación en el aula o el laboratorio serían:

1. Identificar y plantear preguntas que se puedan responder mediante la indagación.

2. Definir y analizar bien el problema por resolver e identificar sus aspectos relevantes.

3. Reunir información bibliográfica para que sirva de evidencia.

4. Formular explicaciones al problema planteado, a partir de la evidencia.

5. Plantear problemas de la vida cotidiana y tocar aspectos históricos relevantes.

6. Diseñar y conducir trabajo de investigación a través de diversas acciones:

a. Generar relaciones hipotéticas y pruebas entre las variables

b. Postular factores causales potenciales.

c. Evaluar la consistencia empírica de la información.

d. Hacer uso de analogías o de la intuición para ayudar a conceptualizar los fenómenos.

e. Formular y manipular modelos físicos y mentales.

f. Utilizar herramientas apropiadas y técnicas para reunir, analizar e interpretar datos.

g. Pensar crítica y lógicamente para desarrollar predicciones, explicaciones y modelos empleando las pruebas.

h. Coordinar los modelos teóricos con la información.

i. Evaluar las explicaciones alcanzadas, con algún modelo científico. 
i. Comunicar hechos y procedimientos científicos en la clase

7. Compartir con otros mediante argumentación lo que se ha aprendido a través de la indagación.

\section{La propuesta del modelo resolución de problemas como investigación}

Algunos autores (Martínez-Aznar e Ibáñez Orcajo, 2005, 2006; Pavón Martínez y Martínez-Aznar, 2014; Rodríguez-Arteche y Martínez-Aznar, 2016a, 2016b) proponen como estrategia de indagación en el aula la metodología de resolución de problemas como investigación (MRPI) (Gil Pérez y Martínez Torregosa, 1983; Martínez Torregosa, 1994 y Ramírez Castro, Gil Pérez). Se fundamenta en la comparación entre cómo resuelven los científicos las situaciones problemáticas que se les presentan en sus investigaciones y el procedimiento que se debe utilizar en las clases de ciencias para que los estudiantes aprendan a resolver sus problemas escolares, favoreciendo así su formación (Martínez-Aznar y Varela Nieto, 2009).

La MRPI se incluye dentro de la indagación ya que los estudiantes trabajan en grupos cooperativos, se enfrentan a situaciones abiertas contextualizadas que deben reformular y definir con precisión, tienen que identificar lo que saben y lo que necesitan conocer, enunciar y contrastar soluciones alternativas y decidir cómo proceder para llegar a una posible solución.

Esta metodología trabaja con problemas abiertos, sin datos y sin solución evidente (situaciones problemáticas o problemas verdaderos). Propone un modelo de resolución que aporta una visión actualizada de la ciencia y pone al alumno en situación de aprendizaje parecida a la de un científico novel (MartínezAznar e lbáñez Orcajo, 2006).

El modelo propuesto está constituido por cinco etapas (véase la tabla 1).

Tabla 1. Etapas de la MRPI

\begin{tabular}{|l|l|l|}
\hline $1 .^{a}$ etapa & $\begin{array}{l}\text { Análisis cualitativo } \\
\text { del problema }\end{array}$ & $\begin{array}{l}\text { Revisar el marco teórico de referencia. } \\
\text { Revisar las posibles concepciones alternativas al respecto cuando } \\
\text { sea necesario introducir nuevos conocimientos. } \\
\text { Reformular el problema en términos operativos (acotarlo de } \\
\text { acuerdo con las intenciones del alumnado) } \\
\text { Indicar las restricciones que se van a introducir para hacer viable la } \\
\text { resolución. }\end{array}$ \\
\hline $2 .^{a}$ etapa & Emisión de hipótesis & $\begin{array}{l}\text { A partir del análisis cualitativo, los estudiantes comienzan a hacer } \\
\text { algún tipo de conjeturas que darán lugar posteriormente a una } \\
\text { emisión de hipótesis. } \\
\text { Reflexionan acerca de las variables que van a influir en el resultado } \\
\text { y la naturaleza de esa influencia. } \\
\text { Las hipótesis orientarán la resolución y luego, permitirán realizar un } \\
\text { análisis riguroso de los resultados. } \\
\text { La ausencia de datos en los enunciados obliga a los alumnos a } \\
\text { pensar en términos de explicaciones provisionales que van a ser } \\
\text { ensayadas. } \\
\text { Las hipótesis emitidas determinan los datos necesarios para la } \\
\text { resolución del problema, en contra del procedimiento de tomar los } \\
\text { datos como punto de partida. }\end{array}$ \\
\hline
\end{tabular}




\begin{tabular}{|c|c|c|}
\hline 3. ${ }^{\circ}$ etapa & $\begin{array}{l}\text { Diseño de estrategias } \\
\text { de resolución }\end{array}$ & $\begin{array}{l}\text { A partir del análisis cualitativo del problema y de las hipótesis } \\
\text { que se han emitido previamente se escogerán las estrategias de } \\
\text { resolución como construcciones tentativas. } \\
\text { Esta etapa permite identificar y controlar las variables que harán } \\
\text { factible resolver el problema reformulado y contrastar las hipótesis } \\
\text { emitidas. } \\
\text { Es el momento de hacer un plan de trabajo donde se indique el } \\
\text { procedimiento por seguir y los materiales necesarios para llevarlo } \\
\text { a cabo. } \\
\text { Las estrategias de resolución en las situaciones problemáticas de } \\
\text { lápiz y papel son, en cierta manera, el equivalente a los diseños } \\
\text { experimentales en los problemas que exigen un contraste práctico } \\
\text { en el laboratorio. } \\
\text { Realizar una planificación correcta sobre las estrategias por utilizar } \\
\text { impide un tratamiento de tipo ensayo/error. }\end{array}$ \\
\hline 4. ${ }^{\circ}$ etapa & $\begin{array}{l}\text { Resolución del } \\
\text { problema }\end{array}$ & $\begin{array}{l}\text { Esta fase se tiene que afrontar de un modo muy flexible, de forma } \\
\text { que los estudiantes puedan "maniobrar" cuando se encuentren con } \\
\text { un obstáculo insuperable. } \\
\text { Siempre que sea posible, se procurará abordar el problema usando } \\
\text { diferentes estrategias. Este procedimiento es extremadamente útil a } \\
\text { la hora de analizar los resultados, pues las coincidencias obtenidas } \\
\text { van a permitir mostrar la coherencia del marco teórico utilizado. } \\
\text { Hacer hincapié en que los alumnos verbalicen los procesos de } \\
\text { resolución que están realizando alejándose, como consecuencia, } \\
\text { de planteamientos lineales carentes de significado teórico. } \\
\text { Verbalizar los procedimientos fomenta la metacognición. }\end{array}$ \\
\hline $5 .^{\circ}$ etapa & $\begin{array}{l}\text { Análisis de } \\
\text { resultados }\end{array}$ & $\begin{array}{l}\text { Es un aspecto esencial del proceso ya que al analizar la } \\
\text { información obtenida a la luz de las hipótesis emitidas se puede } \\
\text { verificar la consistencia interna del proceso realizado. } \\
\text { Un análisis riguroso va a permitir comprobar si la solución obtenida } \\
\text { es correcta o si, por el contrario, es necesario la revisión total o } \\
\text { parcial del proceso. }\end{array}$ \\
\hline
\end{tabular}

Fuente: adaptado de Martínez Aznar y Varela Nieto, (2009).

\section{Consideraciones finales}

La enseñanza tradicional en el laboratorio ha conducido a una comprensión inadecuada de la naturaleza de la ciencia, debido a su enfoque empírico-inductivista del método científico, entendido como un proceso de pasos casi mecánicos: observación, generalización inductiva, hipótesis y verificación, asociado a una imagen objetiva y neutra de la ciencia (Caponi, 2016; Flores Espejo, Caballero Sahelices y Moreira, 2009; Kirschner, 1992).

Más allá del tipo o el contenido abordado, las APL deberían involucrar a los estudiantes en el uso de procedimientos y estrategias lógicos, demostrar las implicaciones de las teorías y las leyes científicas, proporcionar experiencia en hacer buenas preguntas sobre la naturaleza, practicar el reconocimiento de regularidades, simetrías, diversidades y puntos comunes entre las observaciones, reconocer los problemas, comprender los métodos experimentales, organizar e interpretar los datos, comprender la relación de los hechos con la solución de los problemas, planificar experimentos para probar hipótesis y hacer generalizaciones y suposiciones (Kirschner, 1992). 
Así, las actividades prácticas de laboratorio deben promover procesos cognitivos que faciliten la comprensión de un sistema explicativo sobre un hecho o fenómeno más allá de la "simple observación-demostración", y de la manipulación de elementos y materiales de un laboratorio. Este enfoque implica problematizar, promover la elaboración de diseños experimentales, plantear preguntas que cuestionen el sentido común, el análisis de variables observables y teóricas, atender al error experimental, argumentar y reflexionar sobre el proceso y su resultado (metacognición) (Astudillo, 2009; Rivarosa y Astudillo, 2013).

\section{Referencias}

Abrahams, I. (2009). Does Practical Work Really Motivate? A study of the affective value of practical work in secondary school science. International Journal of Science Education, 37 (17), 2335-2353.

Abrahams, I., Reiss, M. y Sharpe, R. (2014). The impact of the "Getting Practical: Improving Practical Work in Science" continuing professional development programme on teachers' ideas and practice in science practical work. Research in Science \& Technological Education, 32(3), 263-280.

Abrahams, I. y Saglam, M. (2010). A study of teachers' views on practical work in secondary schools in England and Wales. International Journal of Science Education, 32(6), 753-768.

Abrahams, I. y Sharpe, R. (2010). Untangling what teachers mean by the motivational value of practical work. School Science Review, 92, $111-115$.

Astudillo, M. (2009). Itinerarios pedagógicos y narrativos: recorrido por una experiencia de formación. En M. Astudillo, R. Carolina y C. Astudillo (eds.), Cuadernos de Prácticas
Educativas. Río Cuarto: Universidad Nacional de Río Cuarto.

Barberà, O. y Valdés, P. (1996). El trabajo práctico en la enseñanza de las ciencias: una revisión. Enseñanza de las Ciencias, 14(3), 365-379.

Barolli, E., Laburú, C. y Guridi, V. (2010). Laboratorio didáctico de ciencias: caminos de investigación. Revista Electrónica de Enseñanza de las Ciencias, 9(1), 88-110.

Bonito, J. (1996). Na Procura da Definição do Conceito de "Actividades Prácticas". Revista Enseñanza de las Ciencias de la Tierra, número extraordinario, 8-12.

Bonito, J. y Trindade, V. (2008). La calidad de la enseñanza y las actividades prácticas de laboratorio: análisis e implicaciones de las actividades alternativas a las propuestas del manual escolar, realizadas por alumnos visitantes de la Universidad de Évora. En A. Calonge, L. Rebollo, M. D. López Carrillo, A. Rodrigo e I. Rábano (eds.), Actas del XV Simposio sobre Enseñanza de la Geología (pp. 23-32). Madrid: Instituto Geológico y Minero de España.

Caamaño, A. (2004). Experiencias, experimentos ilustrativos, ejercicios prácticos e investigaciones: $己$ una clasificación útil de los trabajos prácticos. Alambique. Didáctica de las Ciencias Experimentales, 39.

Caamaño, A. (2007). Los trabajos prácticos en ciencias. En M. Jiménez Aleixandre, A. Caamaño, A. Oñorbe, E. Pedrinaci y A. de Pro Bueno (eds.), Enseñar Ciencias (2. ${ }^{a}$ ed.). Barcelona: Graó.

Cañal de León, P., Del Carmen, L., García Barros, S., Jiménez Aleixandre, M. P., Márquez, C., Martínez Losada, C., ... Sanmartí Puig, N. (201 1). Didáctica de la biología y la geología (volumen 2). Graó. 
Caponi, G. (2016). Conocimiento biológico de un mundo físicamente regido. En R. Gutiérrez Lombardo, J. Martínez Contreras y A. Ponce de León (eds.), Cultura y evolución (pp. 19-28). México D. F.: Centro de Estudios Filosóficos y Sociales Vicente Toledano.

Carrascosa, J., Gil Pérez, D. y Vilches, A. (2006). Papel de la actividad experimental en la educación científica. Caderno Catarinense de Ensino de Física, 23(2), 157-181. Recuperado de https://periodicos.ufsc.br/index.php/fisica/article/ view/6274/12764\%0A

Chavez de Souza, N. y Tauchen, G. (2015). Perercepções e ações docentes no laboratório didático. Investigações Em Ensino de Ciências, 20(3), 164-186.

Correa Sánchez, M. y Valbuena Ussa, E. (2007). Aproximación al estado del arte sobre los trabajos prácticos en la enseñanza de la biología (2004-2006). Revista Virtual EDUCYT, 15, 313-319.

Cortés Gracia, A. y De la Gándara Gómez, M. (2006). La construcción de problemas en el laboratorio durante la formación del profesorado: una experiencia didáctica. Enseñanza de las Ciencias, 25(3), 435-450. Recuperado de https:// goo.gl/bYNKTB.

Del Carmen, L. (2011). El lugar de los trabajos prácticos en la construcción del conocimiento científico en la enseñanza de la biología y la geología. En P. Cañal de León, L. del Carmen Martín, S. García Barros, M. Jiménez Aleixandre, C. Márquez, C. Martínez Losada, ... N. Sanmartí Puig (eds.), Didáctica de la biología y la geología (volumen 2, p. 42). Barcelona: Graó.

Del Carmen, L., Caballer, M., Furió, C., Gómez Crespo, M., Jiménez Aleixandre, M. P., Jorba, J., ... Vilches, A. (2000). La enseñanza y el aprendizaje de las ciencias de la naturaleza en la educacion secundaria. Barcelona: Horsori.

Espinosa Bueno, J., Garritz Ruiz, A., Labastida Piña, D. y Padilla, K. (2010). Indagación. Las habilidades para desarrollarla y promover el aprendizaje. Parte II. El cuestionario y su aplicación. Educación Química, 21 (3), 190-197.

Espinosa Bueno, J., Labastida Piña, D., Padilla, K. y Garritz Ruiz, A. (201 1). Pedagogical content knowledge of inquiry : An instrument to assess it and its application to high school in-service science teachers. US-China Education Review, 8(5), 599-614.

Fernández-Marchesi, N. (2014). Los trabajos prácticos de laboratorio de biología en los libros de texto de ciencias naturales para el nivel secundario utilizados en la ciudad de Ushuaia (tesis de maestría). Facultad de Ingeniería, Universidad Nacional del Centro de la Provincia de Buenos Aires.

Fernández-Marchesi, N., Marcangeli, M. y Romero, C. (2011). Análisis de las estrategias de enseñanza de los docentes de ciencias naturales en dos escuelas 
públicas medias de tierra del fuego. Tecné, Episteme y Didaxis, TED, número extraordinario, 1381-1386.

Flores Espejo, J., Caballero Sahelices, M. y Moreira, M. A. (2009). El laboratorio en la enseñanza de las ciencias: una visión integral en este complejo ambiente de aprendizaje. Revista de Investigación, 33(68), 75-1 11.

García Barros, S., Mondelo Alonso, M. y Martinez Losada, C. (1998). Hacia la innovación de las actividades prácticas desde la formación del profesorado. Enseñanza de las Ciencias, 16(2), 353-366.

Garritz Ruiz, A. (2010). Indagación: las habilidades para desarrollarla y promover el aprendizaje. Educación Química, 21 (2), 190-197.

Garritz Ruiz, A., Labastida Piña, D., Padilla, K. y Espinosa Bueno, J. (2009). El conocimiento didáctico del contenido de la indagación. Un instrumento para capturarlo. Enseñanza de las Ciencias, número extraordinario, 723-727.

Gil Pérez, D. y Martínez Torregosa, J. (1983). A model for problem-solving in accordance with scientific methodology. European Journal of Science Education, 5(4), 447-455.

Hodson, D. (1994). Hacia un enfoque más crítico del trabajo de laboratorio. Enseñanza de las Ciencias, 12(3), 299-313. Recuperado de https://goo.gl/MYyNts

Hofstein, A. y Mamlok-Naaman, R. (2007). The laboratory in science education: the state of the art. Chemistry Education Research and Practice, 8(2), 105-107.

Khan, S. (2007). Model-based inquiries in chemistry. Science Education, 91 (6), 877-905.

Kirschner, P. (1992). Epistemology, practical work and academic skills in science education. Science \& Education, 7 (3), 273-299.
Leite, L. y Figueiroa, A. (2004). Las actividades de laboratorio y la explicación científica en los manuales escolares de ciencias. Alambique. Didáctica de las Ciencias Experimentales, 10(39), 20-30.

Linn, M., Davis, E. y Bell, P. (2004). Internet environments for science education. New Jersey: Lawrence Erlbaum Associate.

Martínez-Aznar, M. M. e Ibáñez Orcajo, I. (2005). Solving problems in genetics. International Journal of Science Education, 27(1), $101-121$.

Martínez-Aznar, M. M. e Ibáñez Orcajo, I. (2006). Resolver situaciones problemáticas en genética para modificar las actitudes relacionadas con la ciencia. Enseñanza de las Ciencias, 24(2), 193-206. Recuperado de https://goo.gl/iHZEit

Martínez-Aznar, M. M. y Varela Nieto, M. P. (2009). La resolución de problemas de energía en la formación inicial de maestros. Enseñanza de las Ciencias, 27(3), 343-360.

Martins, I., Veiga, M. L., Teixeira, F., Tenreiro-Vieira, C., Marques Vieira, R., Rodrigues, A. y Couceiro, F. (2007). Educação em Ciências e Ensino Experimental. Formação de Professores. Colecção Ensino Experimental das Ciências. Lisboa: Ministério da Educação - DGIDC.

Nieda, J. (1994). Algunas minucias sobre los trabajos prácticos en la enseñanza secundaria. Alambique. Didáctica de las Ciencias Experimentales, 2, 15-20.

Pavón Martínez, F. y Martínez-Aznar, M. M. (2014). La metodología de resolución de problemas como investigación (MRPI): una propuesta indagativa para desarrollar la competencia científica en alumnos que cursan un programa de diversificación. Enseñanza de las Ciencias, 32(3), 469-492. 
Ramírez Castro, J., Gil Pérez, D. y Martínez Torregosa, J. (1994). La resolución de problemas de física y de química como investigación. Madrid: Ministerio de Educación y Ciencia.

Rivarosa, A. y Astudillo, C. (2013). Las prácticas científicas y la cultura: una reflexión necesaria para un educador de ciencias. Revista CTS, 8(23), 45-66. Recuperado de https://goo.gl/mpqDyq

Rodríguez-Arteche, I. y Martínez-Aznar, M. M. (2016a). Introducing inquiry-based methodologies during initial secondary education teacher training using an open-ended problem about chemical change. Journal of Chemical Education, 93, 1528-1535.

Rodríguez-Arteche, I. y Martínez-Aznar, M. M. (2016b). La competencia sobre planificación de investigaciones en $4^{\circ}$ de ESO: un estudio de caso. Revista Complutense de Educación, 27(1), 329-351.

Sanmartí Puig, N. (2002). Didáctica de las ciencias en la Educación Secundaria Obligatoria. Madrid: Síntesis.

Saraiva-Neves, M., Caballero Sahelices, M. y Moreira, M. A. (2006). Repensando o papel do trabalho experimental, na aprendizagem da física, em sala de aula um estudo exploratório. Investigações Em Ensino de Ciências, 17 (3), 383-401.

Tamir, P. y García Rovira, M. P. (1992). Características de los ejercicios de prácticas de laboratorio incluidos en los libros de textos de Ciencias utilizados en Cataluña. Enseñanza de las Ciencias, 10(1), 3-12.

\section{Para citar este artículo}

Fernández-Marchesi, N. (2018). Actividades prácticas de laboratorio e indagación en el aula. Tecné, Episteme y Didaxis: TED, 44, 203-218. 